\title{
Perspectives in marine science: a European point of view
}

\author{
K.-G. Barthel \\ Commission of the European Communities, DG XII/D3, MAST Programme; \\ Rue de la Loi 200, B-1049 Brussels
}

\begin{abstract}
Marine research has always been a field in science which was particularly open to, and at times dependent on, international cooperation, and this has become even more obvious during the last decade when issues of global change became central to any discussion. The global nature of scientific and other problems required the development of new concepts and led to the establishment of new structures in research coordination and funding on an international level. In Europe the 12 member European Community often served as a nucleus for larger networks and initiatives (e.g. COST, EUREKA), and in 1989 the EC itself launched a specific programme on marine research and technology (MAST). The various initiatives are not meant to replace national efforts but to complement them - where added value arises from international cooperation, e.g. in global programmes like IGBP, WCRP and their various core projects. The focus of support for these programmes through international funding agencies and networks is not primarily on additional research money but more on structural support and coordination. In contrast, the MAST targeted projects on the North Atlantic margin and the Mediterranean also receive substantial basic support, and are designed to fill gaps left by other international research projects. Both EC and other projects profit from the coordinating measures offered by the EC Commission. A more efficient use of facilities (research vessels, special equipment) can be achieved by having central information services. Well-integrated international projects also require additional efforts in standardization of instrumentation, methods and units, with respect to sampling, sample processing and data treatment. Furthermore, the scope of the task to tackle questions of global change demands the development of new technologies like ROVs, biosensors, automatized sample and data acquisition and treatment, etc. Full exploitation of the results in scientific, political and economical terms is only facilitated through special concertation. Mathematical modelling of ecosystems is still in its infancy and needs further cooperative development in order to provide tools for forecasting and management. Finally, training and exchange of personnel on a European, and possibly wider, level needs to be intensified in order to meet the requirements of modern science.
\end{abstract}

\section{INTRODUCTION}

When considering European, in contrast to national, aspects of marine research, one must take into account that marine science has always been very open to and particularly for field work - also dependent on, international cooperation. Joint action across the borders was, and often still is, based on the personal initiative of individual scientists and only in later years did bilateral institutional and political agreements complement this structure. In more recent years, changing conditions on a global scale have made necessary a research policy which provides (on an international level) the framework to tackle the newly-arising, as well as the already existing, scientific questions. It was and is, understood, as a basic condition, that international concertation 
should only take place where it creates an extra value in addition to national activities (subsidiarity); this means that international concertation does not replace, but complements, national efforts.

From a European point of view, trans-national cooperation of research teams and multidisciplinarity of the research projects must be fostered in order to optimally exploit the existing knowledge and expertise - in view of solving the ever more urgent scientific questions. Therefore, a specific research programme in the field of marine science and technology (MAST) was launched in 1989 by the European Community; which concentrates on basic research and technology aspects as well as improving the research infrastructure. The European Community is in the unique position of having a transnational administration which can thus provide research funding for all teams in an international cooperation out of one hand. This guarantees the necessary synchronization of all teams, usually leads to closer links between them, and thereby increases the efficiency of the work.

The activities in the MAST-I pilot programme were concentrated on research projects complemented by supporting initiatives in some key areas. In order to use the available funds optimally, priority was given to process studies in coastal zones and European shelf seas and on the development of instrumentation. The comparatively good knowledge of principal processes in European waters served as a basis for a number of modelling studies dealing with physical, biological and sedimentological aspects. Developing these modules into composite tools for management purposes and global change forecasting will be the logical continuation of such projects. New topics were taken up in the MAST Programme since 1991 which include deep-sea research and shelf edge processes, and integrated approaches were fostered for the Mediterranean and the North Atlantic.

Besides providing more money for addıtional research, it is also necessary to look tor new ways of organizing and managing large-scale projects, of which quite a number have been launched in recent years on a global or European level, e.g. core projects of the International Geosphere-Biosphere Programme (IGBP) and the World Climate Research Programme (WCRP), and the MAST Targeted Projects in the North Atlantic and the Mediterranean. All these initiatives not only require specific organizational efforts, they also demand an improved flow of information, will deploy highly specialized equipment which might have to be developed in the first place, must also arrive at a standardization of the manifold of methods applied, and will generate a huge amount of data to be evaluated. But smaller projects in coastal research or in pollution studies also need better information services and access to large-scale facilities which can be much better provided on an international than on a national level. This means we need:

- the development of common norms, standards and design guidelines;

- new instrumentation for standard ocean work, as well as specialized applications;

- better access to existing data, and improved means for acquisition and handling of new data;

- tools for hind- and forecasting of ecosystems to approach a better management, in particular of coastal regions,

- advanced training of students and young scientists on an international level;

- the necessary funds on both the national and the international level to implement these international large-scale projects. 
The latter item was purposefully put at the end of the list in order to indicate that, while proper funding is certainly indispensable, it is not sufficient to just provide more money to tackle the tasks which were outlined above. Additional measures need to be taken in order to cope with this new dimension of research. In the following, some examples of actions will be given which have already been started or are planned by the MAST Programme or within the framework of EUROMAR.

\section{STANDARDIZATION}

The need to launch more and more monitoring programmes for coastal regions and regional seas, the trend to large-scale international projects and the use of data sets from different sources for modelling purposes, makes standardization of methods and instrumentation indispensable in order to facilitate the comparison of data and results. It is particularly important in this context to arrive at an initial standardization already at the level of instrument development, in order to avoid too many intercalibration exercises. The EUROMAR project FIESTA (Rules for Field Data Quality Standardization) has concentrated on the industrial aspects of sensor construction and data issues, and produced recommendations for standardizations in the fields of sensor interfaces and sensor quality, equipment installation, operational system control, measurement procedures, data acquisition and distribution, and quality control schemes. These standards, after development and testing for acceptance at relevant institutions, will now be communicated to the European standardization organizations and are planned to be introduced Europe-wide, also in the framework of MAST where applicable.

On the level of methods, more intercalibration is necessary for the time being - and intercalibration exercises dealing with key components in ecosystem research can be supported by the EC MAST Programme. Examples are, activities on "In-situ Measurements of Suspended Matter Characteristics" and "Development of Ocean Tracer Measurement" both with the participation of scientists from several European countries, the USA and Canada. Results of the experiments and consultations will be summarized in a report and implemented in all MAST projects dealing with the respective topics.

If appropriate and of general interest to the scientific public, such reports on EC funded activities are published; as, for example, has a recent manual of sampling and analytical procedures for tidal estuaries. This manual was produced as part of an EC funded project which comprised the majority of major European tidal estuaries. The primary purpose was to enable all partners to acquire comparable data within the project. It is, however, of interest to many researchers in this field and is therefore made generally available. It describes sampling strategies, sampling methods, analytical procedures and data banking, with particular emphasis on situations which are typical in estuaries.

\section{LARGE-SCALE FACILITIES}

Some of the scientific problems outlined above require the use of special large-scale equipment which cannot easily be provided on a national level for financial or logistic reasons. This is particularly so in deep-sea research, where benthic observatories for deployment in several thousand metres depth and drilling vessels for deep-sea geology are needed. The existing research submarines, for instance, are very valuable tools but 
their deployment range is mostly limited in depth and particularly in time: problems which could be overcome by unmanned benthic observatories. Therefore a feasibility study for a deep-sea benthic observatory was started by the EC MAST Programme in 1991, in order to explore the scientific and technical requirements for the construction. It will be particularly valuable for long-term registration of the natural variability of ecosystems and for long-term monitoring of human impacts, e.g. in cases of deep-sea mining or dumping. It could (in a different version) also be used for short-term observations and short-time experiments. The study suggests that functional features to facilitate interdisciplinary research on biological, physical and geochemical processes should optimally include:

Sampling and sensing: - sampling of water, sediments and organisms

- measurement of physical, chemical, biological and geological parameters

- observation of objects, events and working routines

- biological and biogeochemical experiments

Technical requirements: - manipulation of sampling and sensing routines

- camera observations

- sample-storage facilities

- data storage facilities

- high energy capacity

Design principles:

- full automation

- modular principle for easy exchange of modules

- disturbance minimization

- robustness

- temperature resistance

- resistance against aggressive substances

- depth range down to 6000 metres

The realisation of such a project not only depends on the development of new instrumentation to be built into the laboratory, it also requires research on new forms of energy supply, on compact data storage media and on dependable communication links for data transfer and remote control of the benthic observatory itself. These aspects are taken care of in separate research projects within MAST and EUROMAR. Based on this feasibility study, the next step would be the design phase for a deep-sea benthic observatory. It should be able to perform at least a selection of the scientific tasks which were identified in the feasibility study.

\section{THE ACCESS TO EXISTING DATA}

A wealth of data and information on many different kinds of questions already exists throughout the world. Part of it is published; other results were never made known to the scientific public. Even the published results were often evaluated in respect to solving a specific question, but the raw data might hold more information which did not seem to be important or were not of interest to the originator at the time of collection. To sample the same or similar information again would be an enormous waste of energy and scarce financial resources. In the case of long-term data sets, which often cover many years, it would be virtuallv impossible due to the time dimension involved. The problem here is 
that, while the localization of certain data sets is often not possible because their existence is not well-known, they might be very important, e.g. in preparing new projects or for large-scale modelling.

The EC MAST Programme therefore started, as one of its supporting initiatives, the compilation of a "European Directory of Marine Environmental Data" (EDMED), which is to be a catalogue of physical, chemical, biological, geological and meteorological data sets from different laboratories, university departments, institutes, data centres and private companies.

It will cover data sets from the last century, when Challenger made her first voyage, to today. The catalogue will not contain the data themselves, but give a description of their nature and accessibility, as well as a description of the data holding centres. The type of data will include professionally-managed holdings and computer databases, adhoc files and collections of hard-copy tabulations, analogue records, images and samples, such as sedimentary cores and biological samples. This compilation is carried out by a consortium led by the British Oceanographic Data Centre and includes all major national data centres in the EC. Each relevant department or scientist is contacted by the national data centre and requested to provide information on data sets held at this institution. The first release of a European-wide version of EDMED is scheduled for 1993. EDMED will be a PC-based data file (complete with supporting software) which can be searched according to various criteria such as type of data, medium of storage, area of collection, year of collection, data holding centre or data set identification number. Copies will be available to all interested organizations, in particular those who have provided data set descriptions. Thus, a better access to a rich source of information will be given to the scientific community; the exploitation will depend on the initiative of the individual scientist who is interested in using historical data for her or his research.

\section{THE EC MAST POLICY WITH RESPECT TO NEWLY-ACQUIRED DATA}

The attempt by the EC Commission's MAST office to give better access to data is not only limited to historical data but includes newly-acquired data as well. The MAST Programme has established a Data Committee of European data experts, which gives advice on all relevant data issues in marine science. Following this advice, a data policy is implemented in the research contracts between the EC Commission and the research organizations which takes into account the property rights of the scientist as well as the interest of the tax-payer to get good value for money. For MAST projects this means that the participating institutes are asked to provide information about data sets to EDMED, thus continuously updating EDMED in respect to MAST funded research. They are furthermore encouraged to store data in a national or international data centre of their choice, carry out quality checking of their data sets and give access to the data after an appropriate exploitation period.

Storing data in a national or an international data centre has the additional advantage that the data can be managed professionally over a long time, even if the project has been terminated, and that they can at the same time be quality checked according to generally accepted standards which both the originator and other possible users will profit from. After a predetermined time, data will have to become part of the public domain and is then much more accessible in official data centres than in institute holdings. 


\section{INFORMATION SERVICES AND INFRASTRUCTURE}

Cooperation in international projects requires a good level of information and a working and easy-to-use infrastructure. Therefore an electronic bulletin board has been established by the EC Commission, which serves as a centre of information exchange on logistics in marine research. The purpose is not to build up a separate European infrastructure - this will fully remain the responsibility of the national administrations but to improve the flow of information and to give better access to large-scale facilities.

This electronic bulletin board or data bank is part of the European Commission Host Organization (ECHO), a computerized information service of the EC Commission which is located in Luxembourg. In the pilot phase the data bank will give information on research vessels of the EC member countries and on their cruise schedules; it has therefore been named EUROSHIP. Further items to be added later are, e.g. specialized equipment, sea-going containerized laboratories and large-scale research facilities. Currently, EUROSHIP contains data on 84 research vessels from Community member states, irrespective of whether they are privately or publicly owned. It gives information on the owners of the ship, the managing company, technical details of the ship, the planned expeditions, the work to be carried out, the geographic working areas, the chief scientists, the ports-of-call and other cruise details.

The information is available to everybody via electronic link and is meant to aid the planning of research cruises. It can give information on when which ship is available knowledge which is usually not available for ships of other nationalities. Often a complete ship is not needed, in particular for smaller groups, and possibilities for joining research cruises (also on research vessels of other states), are greatly enhanced by the EUROSHIP information service. This system is currently in its testing phase. It can already be accessed via the European Commission Host Organization in Luxembourg and will gradually be extended as outlined above. The scope it will take eventually depends also on the use and the demands by the scientists themselves, and feedback is needed in order to design the information service to be as efficient as possible.

\section{CONCLUSION}

In this contribution some of the aspects of marine science, where international research undertakings produce new requirements in research management, have been outlined. To meet these requirements new services need to be offered and existing structures must be adapted, on a national as well as an international level. Multinational organizations which can make an impact in more than one country and which have the advantage of a multicultural background must play a leading role here. An efficient implementation of research policy by the responsible administration does, however, depend on a regular input from the community of active researchers. The EC MAST office in Brussels is therefore keeping close contact with the scientific community, so as to fulfil this role of strengthening Europe's research infrastructure and improving communications between EC scientists.

The views expressed in this paper are those of the author and do not necessarily reflect those of his institution 